\title{
Comparación de Parámetros para una Selección Apropiada de Herramientas de Simulación de Redes
}

\author{
Manuel A. Calle ${ }^{1}$, José D. Tovar ${ }^{2}$, Yor J. Castaño-Pino ${ }^{3,4}$ y Juan C. Cuéllar 3,4 \\ (1) Jámblico. Carrera 15 \#11-51 Buga, Colombia (e-mail: callemac88@gmail.com) \\ (2) Sainc Ingenieros Constructores S.A. Calle 11 \# 100-121 Piso 2, Cali, Colombia. \\ (e-mail: jdariotv@gmail.com) \\ (3) Facultad de Ingeniería, Departamento de Tecnologías de información y comunicaciones, Universidad \\ Icesi, Cali - Colombia (e-mail: yor.castano@correo.icesi.edu.co) \\ (4) Grupo de investigación i2t, Universidad Icesi, Cali - Colombia (e-mail: jcuellar@icesi.edu.co)
}

Recibido Feb. 19, 2018; Aceptado Abr. 17, 2018; Versión final Jun. 21, 2018, Publicado Dic. 2018

\begin{abstract}
Resumen
El objetivo de este artículo es caracterizar y comparar diferentes herramientas de simulación de red, entregando pautas para realizar una selección apropiada de acuerdo a las necesidades de cada usuario o proyecto. Se definieron una serie de características que debe cumplir una herramienta de simulación, entre las cuales se destacan: el tipo de uso dado a la herramienta, la licencia requerida, curva de aprendizaje requerida, plataforma o sistema operativo que requiere para funcionar, resultados gráficos que entregan, que tecnologías de nivel 2 y 3 soportan, y que tipo de tráfico permite modelar. Los resultados obtenidos después de caracterizar cada herramienta de simulación se presentan en una tabla comparativa que permite al lector elegir la herramienta de simulación según sus necesidades. Los factores más influyentes en la selección de una herramienta de simulación son la curva de aprendizaje y el tipo de licencia.
\end{abstract}

\section{Comparison of Parameters for an Appropriate Selection of Network Simulation Tools}

\begin{abstract}
The goal of this paper is to characterize and compare different network simulators, to establish the most appropriate network simulation tool, based on user needs. A series of useful parameters in network simulation for each analyzed tool, such as the type of use, the type of license required, the learning curve, the operative system required, the type of graphic results, the technologies in level 2 and 3 available, and type of network traffic allowed to simulate are defined. This information was summarized in a matrix that links each simulator and the relevant characteristics. The table allows any user to choose a network simulator, considering its context and the simulator goal. The most relevant factors for selecting a simulation tool is the learning curve and the type of license.
\end{abstract}




\section{INTRODUCCIÓN}

La simulación no es un concepto nuevo, siempre se ha buscado la manera de evaluar sistemas complejos y tal como define Phillips (2007), la simulación es la ejecución de un modelo representado por un programa de computadora, que permite recrear entornos de red, ahorrando tiempo y dinero. En el área de investigación de telecomunicaciones es muy costoso desplegar una implementación de una red de manera completa, con múltiples computadores, dispositivos de interconectividad y enlaces con el fin de verificar y analizar el desempeño de protocolos o algoritmos de red. La simulación en este sentido, permite el ahorro de tiempo y dinero para que sea posible el diseño de redes complejas a partir de módulos simples. Sin embargo, no solo en el mundo de las redes de comunicaciones es útil simular, este concepto aplica a diferentes ciencias e ingenierías, entre otros campos (Pan y Jain, 2008).

Con la simulación y en particular, con las herramientas de simulación se pueden evaluar, probar y realizar análisis de desempeño de protocolos, dispositivos o esquemas de red e incluso medir la calidad de la experiencia (Cuellar et al., 2014). Las herramientas de simulación han sido ampliamente usadas para fortalecer los procesos de enseñanza-aprendizaje en universidades y en diferentes grupos de investigación. En las universidades se suelen usar para enseñar los fundamentos de las redes de comunicación, la configuración de los dispositivos de red e incluso como mecanismos de preparación para certificaciones profesionales (Goldstein et al., 2005). En el ámbito investigativo, como presenta Cuellar (2011) los simuladores son usados para evaluar el comportamiento de múltiples parámetros de red, como retardo, variación del retardo (Jitter), entre otros, en una red; lo cual permite realizar comparaciones entre diferentes protocolos y así proponer mejoras para aumentar el desempeño de las redes.

De este modo, Goldstein, et al., (2005) plantean el uso de herramientas de simulación de red como parte de un método pedagógico de enseñanza, que permita a los estudiantes entender los diferentes conceptos de una manera más clara, sencilla y representada de una manera tangible, basándose en la premisa de que los estudiantes aprenden más fácil, si disfrutan usando una herramienta educativa. Es así como Perez-Hardy (2003) expone diferentes vías en las que un simulador de red puede ser usado para enseñar a los estudiantes los conceptos básicos, avanzados, el diseño y principios de desempeño. En la academia, diferentes autores abordan el tema, tal como muestran Weingartner et al. (2009), quienes presentan los conceptos de simulación y hacen una comparación entre diferentes herramientas. Los resultados obtenidos muestran la experiencia que los usuarios experimentaron con el uso de las diferentes herramientas, como también, si se presentó la necesidad de realizar cambios en el código fuente de las mismas. Además, muestran como en la mayoría de los casos, usar las herramientas de simulación no es un proceso dispendioso, pero algunas poseen limitaciones para simular entornos complejos o no estandarizados.

Actualmente, existen gran cantidad de simuladores de red, cada uno de ellos con características y capacidades diferentes, lo cual dificulta la selección de la herramienta a usar según la necesidad o contexto. En este artículo se abordan diez de las herramientas más conocidas y usadas para la simulación de redes, entre las cuales se encuentran Omnet, Opnet, Ns3, Gns3, Real, Qualnet, J-sim, entre otras. Con base en lo anterior, el objetivo del artículo es presentar las herramientas de simulación más usadas en el ámbito investigativo. Con el fin de que el usuario final tenga la fundamentación necesaria para elegir la herramienta que mejor se adapte a sus necesidades, con base en parámetros cualitativos analizados en múltiples referencias y como un aporte a lo trabajado por García et al. (2011).

\section{PARÁMETROS A ANALIZAR PARA ELEGIR UNA HERRAMIENTA DE SIMULACIÓN}

Los parámetros que se presentan en esta sección son útiles para comparar las diferentes herramientas de simulación sobre una base homogénea, lo que facilita notablemente el proceso de selección. Al final del documento, la Tabla 1 corresponde a una matriz donde para cada una de las diferentes herramientas de simulación analizadas, se presenta el valor de cada uno de los parámetros aquí descritos. La tabla 1, por lo tanto, se constituye en una herramienta rápida de comparación y selección. En la siguiente sección no solo se describe cada parámetro, con base en la revisión bibliográfica realizada, también se establecen los criterios de medición respectivos.

\section{Uso investigativo}

Este parámetro tiene como finalidad establecer niveles para comparar el uso de las herramientas de simulación en áreas académicas e investigativas. Esto con el fin de explorar los aportes que han logrado las diferentes comunidades, respecto al uso de las herramientas y hacer un primer acercamiento que permita identificar las principales implementaciones de red en que son usadas. Los niveles de medida para constituir puntos de comparación entre el uso investigativo de un simulador de red u otro son los siguientes: (i) alto, indica que la herramienta de simulación ha sido utilizada en al menos cinco proyectos de investigación en las 
que se muestran implementaciones desarrolladas con dicha herramienta; (ii) medio, cataloga las herramientas de simulación de red que fueron usadas entre dos y cinco veces en la comunidad investigativa, pero debido a algunas características o especificaciones técnicas de las herramientas, no han logrado la proliferación deseada; y (iii) bajo, indica poco uso investigativo del simulador, debido a las limitaciones de sus características, por lo cual las referenciasen los grupos de investigación es menor a dos proyectos de investigación.

\section{Tipo de licencia}

Este parámetro define el tipo de licencia de la herramienta, que, para efectos prácticos, dependiendo del tipo de licencia, la herramienta puede tener habilitada todas sus funciones o un grupo de éstas, esto también puede ir ligado con el valor que se cobre por la licencia. Con base en lo anterior la forma de categorizar los tipos de licencias son Libre y Comercial. En cuanto a la primera, se busca evaluar si las diferentes herramientas de simulación cumplen con las características definidas por la fundación para el software libre, donde se plantea que este no es necesariamente es una herramienta gratuita, lo que la hace libre es la posibilidad de que los usuarios puedan editarla, copiarla, ejecutarla, distribuirla, estudiarla y mejorarla. Desde la perspectiva de la Free Software Fundation y la GNU operations system, los usuarios de programas libres tienen cuatro libertades esenciales: la libertad de ejecutar el programa para cualquier propósito; la libertad para estudiar cómo funciona el programa y adecuarlo a la forma que cada usuario desee; la libertad de distribuir el código de un usuario a otro; y la libertad de distribución del código modificado a otros. El software libre debe cumplir estos cuatro acuerdos y, en caso de que se cobre algún costo por un programa, esto no inhibe que el programa pueda ser gratuito o utilizado según las mismas pautas. En cuanto a las comerciales serán las licencias que tienen restricciones para el usuario, teniendo en cuenta que su comercialización, costo, duración de uso, edición y libertad de permisos, es controlada y definida por los propietarios que comercialicen la herramienta.

\section{Curva de aprendizaje}

Por medio de este parámetro, se busca catalogar el nivel de exigencia que requiere la herramienta para lograr un manejo adecuado de la misma. Este parámetro es sumamente importante y considera diversos aspectos esenciales para medir la herramienta en este sentido, los cuales son: a) conocimientos previos, puesto que el contexto son las redes de comunicación, es de vital importancia conocer aspectos básicos en lo referente a protocolos y estándares de red para enfrentarse a una herramienta potente, además las herramientas de alto rendimiento permiten la introducción de código, por lo que también es importante tener claro los conceptos básicos en programación; y b) uso didáctico de la herramienta, existe gran variedad de simuladores que en cuanto a interacción son sumamente amigables con el usuario, ya que permiten configurar elementos de red de una manera intuitiva, permitiendo observar solo las características básicas del contexto de red; por esta razón, son usados como herramientas didácticas para la iniciación de los estudiantes en los cursos de redes.

De acuerdo con las características planteadas, los niveles definidos para efectuar la medición de este parámetro en cada uno de los simuladores que se presentarán son los siguientes: (i) alto, herramientas en las que es muy importante una fundamentación solida de conocimientos avanzados en redes y programación, puesto que su manejo exige programación de los dispositivos por modificación de su código fuente; (ii) medio, herramientas que no requieren una gran cantidad de conocimientos previos en programación o redes y donde toda la carga de aprendizaje se centra en la configuración de los dispositivos de red mediante comandos de consola; y (iii) bajo, herramientas de uso didáctico para la enseñanza, en las que la interacción con el usuario hace que la configuración de simulaciones de red se realice de manera intuitiva.

\section{Plataformas que lo soportan}

Este parámetro es descriptivo. Lista los diferentes sistemas operativos en los cuales la herramienta puede correr sin ningún problema. Los sistemas operativos que se tendrán en cuenta serán WINDOWS, LINUX, MAC OS, SOLARIS.

\section{Interfaz gráfica de la herramienta}

Con este parámetro se busca definir el acercamiento que tiene la herramienta con el usuario y las facilidades que le presta. La medida de este se tendrá en cuenta basándose en tres rangos, así: (i) alto, nivel de programación mínimo, ya que la herramienta tiene la disposición de trabajar desde todas sus perspectivas con una interfaz gráfica; (ii) medio, implementa interfaz gráfica que facilita su uso, pero de forma limitada; algunas de sus implementaciones deben definirse mediante programación; y (iii) bajo, no cuenta con interfaz gráfica o no es muy amigable con el usuario, lo cual implica la programación de cada elemento dentro de una simulación para su ejecución final. 


\section{Graficas de resultados}

Las herramientas de simulación se utilizan para recrear el funcionamiento de la red lo más real posible. Para esto es necesario realizar medidas de ciertas variables de red, con el fin de analizar los datos posteriormente y así, comprender el comportamiento de la red, ante diferentes eventos o posibles configuraciones. Ahora una manera de interpretar y analizar los datos de las variables medidas es graficándolos de diversas formas y dependiendo de qué tan potente o amigable sea la herramienta de simulación para realizar esta tarea se han definido los siguientes rangos: (i) buena, la herramienta posee extensiones o módulos propios para la generación graficas estadísticas las cuales pueden ser manipuladas desde el mismo simulador o exportándolas a un procesador especializado; (ii) aceptable, simuladores que pueden generar datos estadísticos pero que necesitan de una herramienta externa, para generarlos, procesarlos adecuadamente y presentar la información ordenada al usuario; y (iii) limitada, que no cuenta con un módulo propio o extensión para la generación de gráficos, la información estadística puede estar representada en archivos de texto que necesitan de herramientas diferentes a la de simulación para su organización y presentación.

\section{Tecnologías de nivel 2 y 3 que soporta}

Parámetro de índole descriptivo en el cual se listan los protocolos de nivel 2, 3, 4 y 7 del Modelo OSI que la herramienta soporta. Algunas herramientas no soportan todos los protocolos por lo cual se hace necesaria su implementación generando código o adaptando componentes preexistentes. De este modo se quiere clasificar los simuladores de acuerdo con la variedad de protocolos que permite simular, basándose en los siguientes criterios: (i) alto, herramientas que permiten la implementación de gran cantidad de protocolos de red, ya que poseen módulos propios con la arquitectura necesaria para que sean soportados y desplegados de manera correcta, con el fin de acercarse a implementaciones reales; (ii) medio, simuladores que no permiten realizar implementaciones de un gran número de protocolos puesto que no posee los módulos necesarios o en su defecto es necesario modificar el código fuente de algún modulo para lograr simular el protocolo deseado y (iii) bajo, herramientas que no poseen los módulos de los protocolos desarrollados o en su defecto es necesario modificar conseguir los módulos por aparte.

\section{Tráfico que permite modelar}

Este es un parámetro muy importante para las herramientas de simulación ya que permite establecer qué tipo de aplicaciones, servicios o protocolos la herramienta está en capacidad de simular. Esto va muy ligado con el comportamiento de aplicación, en el sentido de cómo se generan los datos a utilizar en la simulación, lo cuales están ligados a una distribución estadística que se puede parametrizar en la herramienta, tanto para las aplicaciones que dispone la herramienta como para aplicaciones que el usuario pueda ajustar dependiendo de sus necesidades. Por ello, se debe conocer de antemano que ofrece la herramienta para realizar esta labor que puede ser medida de acuerdo con los siguientes criterios: (i) alto, herramientas con la capacidad de generar tráfico de gran variedad de aplicaciones de acuerdo con diferentes distribuciones estadísticas y también son capaces de recibir la inyección de tráfico proveniente de analizadores de tráfico (Sniffers) para realizar análisis estadísticos; (ii) medio, herramienta que permite generar tráfico de las aplicaciones más comunes, donde las distribuciones estadísticas se pueden configurar de manera básica; y (iii) nulo, herramienta en las que no es posible configurar distribuciones de tráfico que permitan análisis académicos profundos o en su defecto no cuentan con módulos o extensiones para realizar esta labor.

\section{Aplicaciones en telecomunicaciones}

Dado que las capacidades de simulación son diferentes para cada software, es importante resumir las aplicaciones para la cual es útil cada simulador. Mediante este parámetro se pretende dar a conocer de forma puntual y resumida el uso de cada herramienta de simulación en el área de telecomunicaciones.

\section{Ventaja competitiva}

Este parámetro se concentra en destacar el(los) punto(s) fuerte(s) de cada simulador. De esta forma, se puede conocer el enfoque para el cual fue desarrollado el simulador y así mismo discriminar entre las herramientas usando un criterio de selección basado en el valor agregado de cada una.

\section{ANÁLISIS COMPARATIVO DE LAS HERRAMIENTAS DE SIMULACIÓN}

Una vez definidos los parámetros de comparación, se procederá a evaluar cada uno, en las diferentes herramientas de simulación, con el fin de establecer un cuadro comparativo (Tabla 1) que le permita al usuario visualizar de una manera clara las ventajas y desventajas de elegir una herramienta u otra. 


\section{Herramienta Ns2}

Sarkar y Goel (2008) indican que NS2 es una de las herramientas de código abierto más confiables y completas para la disposición e implementación de redes. Sus posibilidades de uso, disponibilidad al público y las características del software, permiten que gran cantidad de usuarios desplieguen implementaciones sobre esta plataforma, convirtiéndola en una de las herramientas preferidas por la comunidad investigativa (Bateman y Bhatti, 2010, y Fan y Taoshen, 2009). Continuando con uno de los elementos que ayudan al uso de la herramienta, se presenta la libertad de disposición que tiene el simulador (Bateman y Bhatti, 2010), argumentando la disponibilidad del código fuente tanto para su inspección, modificación y la libertad para la aplicación de cualquier usuario. Esto ha promovido generación de módulos entre de la comunidad, que permiten la creación de nuevos protocolos y sistemas para ser simulados. A su vez la característica de licencia libre que presenta la herramienta, ha permitido expresar las preocupaciones sobre la herramienta y la influencia positiva que esta ha causado.

Para centralizar una característica más del simulador NS2, es preciso divisar sus dos niveles de simulación posibles explicados en Fan y Taoshen (2009) y Qun y Jun (2008), donde una se basa en la configuración y construcción de Otcl referido a programación orientada a Objetos con extensión tcl., opción en la cual se pueden utilizar algunos elementos de redes existentes en la herramienta para realizar una simulación escribiendo scripts en Otcl sin la necesidad de modificar NS2. Por otro lado, se tiene la programación en lenguaje $\mathrm{C}++$ y Otcl, la cual es utilizada cuando los módulos utilizados para las implementaciones no existen, lo que obliga a actualizar la herramienta con los nuevos elementos y, en ciertas circunstancias, se agregará una clase programada en $\mathrm{C}++$ y una clase en Otcl y seguido, programar un script Otcl para la implementación de la simulación. Esto genera la necesidad de un gran conocimiento previo para el uso de la herramienta, una evidencia de esta necesidad se da en López et al., (2014) y García et al. (2015) donde se menciona el uso simultáneo de dos lenguajes de programación $(\mathrm{C}++$ y $\mathrm{TCL}$ ) para simular y estudiar el rendimiento de algoritmos de balanceo, sobre redes GMPLS usando NS-2. Adicional a esto, como se muestra en Marques et al. (2009), es necesario para la correcta ejecución de la herramienta tener experiencia, lo que lleva a la necesidad de usar la herramienta por largos periodos de tiempo para lograr su implementación eficaz; entonces, NS2 se cataloga con un nivel alto en su curva de aprendizaje.

Dado que el código fuente de la herramienta se encuentra disponible para modificación y crecimiento del simulador, esto representa una fuerte ventaja en el ámbito de la investigación. Esta ventaja se puede ver resaltada en Li et al., (2017) en donde se usa esta herramienta para simular aplicaciones seguras en redes de área vehicular; y en Ben Fraj et al., (2018) quienes evalúan el desempeño de los recientes protocolos anticolisión, implementando dos nuevos módulos de simulación RFID, que pueden ser usados en el área de internet de las cosas. En cuanto al valor agregado del simulador NS2, es importante resaltar que este fue diseñado para investigación en redes y para proveer soporte para simulaciones TCP, y protocolos de envío múltiple sobre redes cableadas e inalámbricas. Además, de esto se enfoca en el sector educativo como herramienta de simulación para enseñanza e investigación.

NS2, es un simulador con limitadas características gráficas. Como se planteaba, está orientado a programación como se presenta en Marques et al. (2009); aunque se han hecho intentos de simulación gráfica para aprovechar la potencia de la herramienta donde sólo se ha intentado graficar las rutas de los paquetes como explican Qun y Jun (2008) implementando así la herramienta NAM. Por lo tanto, NS2 es una herramienta que se encuentra catalogada con un bajo nivel de características gráficas. Aunque el software no contiene una herramienta que retorne gráficas de una manera eficiente, existen diversas herramientas que permiten hacerlo, lo cual minimiza el problema y, permite aprovechar todos los valores que arroja el simulador de las tecnologías manejadas a nivel 2 y 3 de OSI, como lo describen las prácticas presentadas por Shin et al. (2009), donde definen tecnologías que la herramienta puede simular TCP/IP, UDP, FTP, RTP, SRN, GPRS, IPv6 móvil, RSRV, MPLS así como redes Ah Hoc, WLAN, Mobile-IP, UMTS y redes inalámbricas. Finalmente, otro aspecto positivo de la herramienta de simulación según Bateman y Bhatti (2010) está relacionado con la diversidad de plataformas que la soportan, dentro de las que se destacan FreeBSD, Linux, SunOs, Solaris, Windows y Mac OS X.

\section{EstiNet}

EstiNet es un simulador y emulador de red, que se originó de NCTUns, el cual fue usado ampliamente en investigaciones relacionadas con redes de comunicaciones desde el año 2002 hasta el 2011. NCTuns se volvió un software comercial en 2011, y se renombro como EstiNet. Esta nueva versión del software comercial incluye la capa física, de control de acceso, de red, de transporte y de aplicación. Adicional a esto, incluye una interfaz gráfica mediante la cual se puede construir y depurar la simulación, asi como observar los resultados de la misma. Según el sitio web oficial, entre sus tres beneficios principales están, (i) la integración directa con la pila de protocolos del núcleo TCP/IP y UDP/IP de Linux, lo cual permite obtener un comportamiento real en ambientes de simulación; (ii) compatibilidad con aplicaciones de red Linux del mundo 
real, lo cual permite probar y configurar simulaciones de red en aplicaciones del mundo real; y (iii) permite interactuar con dispositivos de red del mundo real. En la actualidad EstiNet se encuentra en la versión 9.0, pero ya en anteriores versiones ha demostrado sus capacidades de simulación y emulación, Wang et al. (2013) comparan EstiNet con simuladores de red como NS-3 y Mininet, con respecto a los parámetros de repetitividad de los resultados, escalabilidad, correctitud de los resultados y soporte de interfaz gráfico, de lo cual concluyen que EstiNet tiene mejores resultados en cuanto a desempeño y escalabilidad.

Kitanov y Janevski, (2013) evaluaron las posibilidades, capacidades y características de EstiNet para simular y emular el comportamiento de las redes LTE, para esto diseñaron un simple escenario con al menos un usuario LTE conectado a internet, y posteriormente evaluaron el desempeño para trafico TCP y UDP de descarga. Finalmente, concluyeron que EstiNet es una herramienta adecuada para simular y emular el comportamiento de las redes LTE. También, Batish y Dhiman (2012), usaron la versión 7 de Estinet, para un estudio comparativo de los protocolos AODV [Ad Hoc On-Demand Distance Vector], DSDV [DestinationSequenced Distance Vector Routing] y DSR [Dynamic Source Routing], dicha simulación se logró gracias a la interfaz gráfica del software, la cual provee una forma intuitiva de construir los escenarios de simulación. Por otro lado, la herramienta de simulación EstiNet, con todas sus propiedades, tiene una limitante, ya que sólo se puede implementar en sistema operativo LINUX, tal como lo especifica el sitio web oficial, ya que el simulador aprovecha la pila de protocolos TCP/IP de Linux para lograr alta confiabilidad en la respuesta de las simulaciones. Gracias al gran desarrollo que ha tenido la herramienta de simulación EstiNet, el software tiene la posibilidad de simular, características y elementos de redes alámbricas e inalámbricas como enrutadores, conmutadores, y dispositivos finales; protocolos de red como árbol de expansión, algoritmos de enrutamiento como OSPF y RIP; también permite simular puntos de acceso inalámbricos, bajo los protocolos $802.11 \mathrm{a} / \mathrm{g} / \mathrm{n}$, modelos de canales, entre otros.

Finalmente, cabe resaltar que este simulador se enfoca en el sector educativo como herramienta de simulación para enseñanza e investigación. Además de esto, como valor agregado, cuenta con una interfaz gráfica amigable y con tres características que lo diferencian ampliamente de los demás simuladores: (i) integra la pila de protocolos del núcleo de linux, lo cual permite obtener un comportamiento real de los protocolos de las capas tres y cuatro simuladas; (ii) es compatible con aplicaciones de red reales del sistema operativo linux, lo cual permite desarrollar y probar aplicaciones y funcionalidades en entornos simulados garantizando un igual comportamiento a cuando se despliegue la aplicación en un dispositivo de red real con sistema operativo linux; y finalmente, (iii) interactúa con dispositivos de red reales, mediante la función de emulación un dispositivo o red simulada puede interactuar con una red o un dispositivo del mundo real.

\section{Opnet modeler}

Esta es una herramienta de simulación que permite flexibilidad y escalabilidad en modelos jerárquicos los cuales representan estructuras de redes reales y puede ser implementada en sistemas operativos tipo Unix o Windows. Dichos modelos, están divididos en 3 dominios, denominados Red, Nodo y Procesos, los cuales están escritos en $\mathrm{C}_{++}$y poseen su propio editor como se explica en Zirong y Huaxin (2009). Esta herramienta de modelado de redes cuenta con dos factores diferenciales importantes: (i) un motor de simulación de eventos con alto desempeño, lo cual permite ejecutar rápidos análisis de impacto y comportamiento en modelos de redes, y (ii) cuenta con una amplia biblioteca de modelos de red compatibles y listos para ser usados, o modificados para generar modelos de aplicaciones y protocolos personalizados, lo cual podría resultar en una reducción de los tiempos de simulación. Además de esto, no se centra en un solo sector de aplicación, en este caso, este simulador puede ser usado en la industria, ya que permite simular comportamientos de extremo a extremo en diferentes diseños tecnológicos, también es usado en la academia para investigación y desarrollo de redes, así como la generación de tecnologías o protocolos de comunicación inalámbricos.

Gracias a las numerosas ventajas y debido a la poderosa interfaz gráfica y estadística que maneja, el uso por parte de grupos académicos es alto, puesto que la manera de simular es muy intuitiva, pero necesita de gran cantidad de conocimientos previos en redes y programación para enfrentarse a la herramienta, por lo cual la curva de aprendizaje de OPNET es alta. De otro lado, aunque se debe aclarar que la licencia del simulador es comercial, existe una versión de este software que no genera cargos económicos para utilizarlo en actividades netamente académicas. Puesto que OPNET proporciona los mecanismos necesarios para el desarrollo fluido de una simulación, permitiendo arrastrar componentes para conformar topologías de red, Xia et al. (2008) configuraron una solución de MPLS VPN activando los diferentes parámetros desde un cuadro de diálogo que ofrece una lista de ellos. Otro escenario donde se usó el poder de Opnet para crear escenarios de simulación de redes se muestra en García et al., (2014), ellos analizaron la calidad de servicio que perciben los usuarios Wimax cuando se interconectan a un Core MPLS. Para esto, plantearon dos escenarios modificando los parámetros de calidad de servicio. Como conclusión, determinaron que la asociación de la conmutación de etiquetas junto con los servicios diferenciados son factores claves que permiten elevar el rendimiento de la red. 
Para el estudio de los resultados de simulación en OPNET, este cuenta con características que permiten realizar esta tarea. El servicio de análisis consiste en mostrar la información de los resultados de simulación en forma de grafo, presentados dentro de áreas rectangulares llamadas paneles de análisis, los cuales tienen propósitos específicos para presentar la información o transformar la existente. Ahora, por el lado de las tecnologías de nivel 2 y 3 que la herramienta soporta, se tienen diversas implementaciones al respecto. Para empezar Hammoodi et al. (2009) realizaron las implementaciones de tecnologías WSN (Wireless Network Sensor) aunque el simulador no soporta modelos de energía, importantes para este tipo de redes. Adicional a esto, Kulgachev y Jasani (2010), evalúan el desempeño del protocolo RTS/CTS en redes con tecnologías como $802.11 \mathrm{~g}$ y $802.11 \mathrm{~b}$. Finalmente, Shehab et al. (2018a, 2018b) describen y analizan una propuesta de esquema eficiente para un sistema de video por demanda entre pares (P2P VoD), de lo cual concluyen que los resultados de simulación muestran que el modelo propuesto puede mejorar eficientemente tanto la carga del servidor como el retraso o latencia de la emisión inicial.

\section{Omnet++}

Esta es una herramienta de simulación de eventos discretos. Tal como específica Varga (2001), la motivación para el desarrollo de OMNET fue producir una herramienta poderosa de código abierto que pueda ser usada por la academia, educación o investigación y que fuera una alternativa a las herramientas comerciales para ser implementada en sistemas tipo Unix, MAC y Windows; incluso haciendo uso de Cygwin o el compilador de Microsoft Visual $\mathrm{C}_{++}$es posible portar la herramienta en otros sistemas con menor esfuerzo. Este software ha estado disponible desde septiembre de 1997 y, fuera de las descargas anónimas, alrededor de 40 universidades a nivel mundial han obtenido la herramienta para su uso, indicando que las áreas de aplicación en la que se utilizará van desde tecnologías móviles, inalámbricas hasta ATM y redes ópticas, realizando proyectos relacionados con el desarrollo de modelos de TCP/IP en la universidad Karlsruhe. El proyecto "OMNET remoto" para el manejo de simulaciones remotas e investigaciones de ejecución en paralelo usando el método de sincronización estática. Esta herramienta se conforma por módulos escritos en $\mathrm{C}++$ que se comunican entre sí por paso de mensajes, donde módulos simples, pueden conformar módulos compuestos y los niveles jerárquicos no tienen límites. Se debe tener en cuenta, que a pesar de que la herramienta de simulación presenta muchos beneficios, los módulos que la conforman no están del todo desarrollados, lo cual implica que los programadores deban modificar el código existente o realizar implementaciones de módulos nuevos para cubrir componentes de red aún no especificados dentro del paquete OMNET; esto hace que la curva de aprendizaje de la herramienta sea alta ya que modificar el código fuente de los componentes de red no es una tarea sencilla.

En cuanto a la interfaz gráfica que ofrece la herramienta, como ya se mencionó las características de depuración son muy buenas, adicional a esto, Lessmann et al. (2008) destacan que OMNET es un simulador con visualización en línea, lo que permite que los usuarios puedan pausar la simulación e inspeccionar o cambiar los valores de los módulos que conforman una red. Incluso, es posible cambiar la apariencia de los nodos y datos que arroja como resultado para ser analizados con herramientas estadísticas que se pueden encontrar en el repositorio de OMNET en la web. Sin embargo, si el módulo no está desarrollado en alguna librería de OMNET, será necesario generarlo mediante código y la interfaz gráfica en este caso se reduce a un editor de texto. Esto último se puede evidenciar en Kucerak y Chlumsky (2016), donde se describe una nueva implementación de protocolo SIP, que permita complementar la herramienta, ya que la implementación actual del protocolo SIP está incompleta. Es importante aclarar que no solo se pueden crear nuevos módulos, también se puede utilizar este simulador para crear otras herramientas, tal como Merzoug et al (2018), quienes desarrollaron una plataforma para simular y modelar sistemas de cómputo en la nube, usando el poder de simulación y el código disponible de OMNET.

Con lo relacionado a las tecnologías de nivel 2 y nivel 3 que soporta la herramienta, diversas publicaciones muestran los diferentes protocolos e implementaciones realizadas. En primera instancia, Rhee et al. (2009) muestran el diseño de un esquema de enrutamiento específico que pueda proporcionar los niveles de QoS basados en los acuerdos de servicio y administración de recursos con el suscriptor. También Morales et al. (2013) presentan que, en la mayor cantidad de los casos, el encolamiento LLQ (LowLatencyQueuing) es más propicio de aplicar, ya que permite a todas las aplicaciones llegar a su destino final y genera tiempos efectivos para las aplicaciones más sensibles a fallas. Finalmente, Zhu et al. (2008), presentan el diseño de un dispositivo de QoS para descartar paquetes de flujos que no se vean tan alterados por la pérdida de los mismos, evitando, que el descarte se haga aleatoriamente sobre cualquier flujo, inyectando para esto, tráfico MPEG, H323 y MP3 midiendo el retardo de la transmisión de cada flujo y la tasa de paquetes perdidos, generando por medio de gráficas estadísticas detalladas. Adicional a esto, Chen et al. (2010) presentan un caso interesante de televisión digital para usuarios residenciales con conexiones a internet de manera asíncrona y usando NAT, con el fin de que compartan datos directamente con otro. La simulación realizada en OMNET contó con 1000 pares los cuales al iniciar se unieron a un pseudo sistema P2PTV en tan sólo cinco minutos, obteniendo resultados que mostraron que la implementación incrementa la tasa de compartimiento entre pares que usan NAT un $50 \%$, lo cual hace más escalable la arquitectura y reduce alrededor de 20 a 24\% el tiempo de descarga de paquetes. 


\section{Herramienta Ns-3}

Esta herramienta es un simulador de eventos discretos de red, cuyos objetivos principales son lograr un mayor énfasis en los niveles 2 y 4 del modelo OSI y que su uso sea principalmente educativo, aunque no se encuentran publicaciones referentes a la herramienta tan fácilmente. En un principio la compatibilidad con NS-2 no es un objetivo del proyecto, por lo que NS-3 no es una actualización de NS-2 sino un proyecto diferente. Esta herramienta es de código abierto y permite la inclusión de otro software de código abierto, escalable modular y emulador.

La interfaz gráfica de esta herramienta, como indican Henderson et al. (2006) soporta algunas formas de animación visual para todo o parte de una simulación. Es una herramienta útil para depurar y mostrar la simulación a terceros. Usa NAM para la animación del escenario de red, siendo parte de NS-2 desde un principio y se ha logrado hacer un extendido en ambientes Wireless. Ahora, para la generación de resultados, la publicación indica que NS-3 proporciona objetos de soporte para facilitar la recolección de datos durante la ejecución de la simulación, estando incluida desde NS-2 la característica. NS-3 se extiende incluyendo objetos para histogramas, rastreo, funciones de distribución y secuencias versus tiempo. En lo relacionado con la usabilidad y adaptabilidad, NS-3 está escrito solo en $\mathrm{C}_{++}$lo que hace que sea más fácil de depurar (Brugge et al,. 2010). Las plataformas que soportan esta herramienta tanto de escritorio como de servidor son i386, x86-64, Linux, OS X, freeBSD, Solaris y Windows. Respecto a las tecnologías que soporta la herramienta, NS-3 permite simulaciones sobre IPv4 e IPv6, Wireless como Wifi, Wimax y algoritmos de enrutamiento como OLSR [Optimized Link State Routing] y AODV [Ad-hoc On-demand Distance Vector Routing] (Wang y Liu, 2010).

Finalmente, se enfoca en el sector educativo como herramienta de simulación para enseñanza e investigación, además, como factor diferenciador, permite realizar un amplio conjunto de simulaciones en redes basadas en IP y en no IP. También, cuenta con una gran comunidad de desarrolladores que constantemente liberan versiones estables, específicamente cada tres meses, lo cual implica la inclusión de nuevos modelos, documentados, validados y con soporte para realizar simulaciones en escenarios actualizados y con los últimos estándares de telecomunicaciones.

\section{Simulador Gns3}

Software gráfico de simulación de red que permite la emulación de redes complejas, trabajando similar a reconocidos emuladores como Vmware o Virtual Box, emulando los IOS de los dispositivos de interconectividad de Cisco. Dynamips es el núcleo de este software y es el que permite esta tarea. Adicionalmente la herramienta de simulación corre en ambientes Linux, Windows y Mac. En cuanto a la licencia de esta herramienta, es de libre descarga, pero necesita de las imágenes de los dispositivos Cisco para emular el comportamiento de estos y dichas imágenes se deben adquirir directamente con el fabricante. GNS3 no toma el lugar de un enrutador real, pero es una herramienta para el aprendizaje y preparación para certificaciones cisco como CCNA [Cisco Certified Network Associate], CCNP [Cisco Certified Network Professional] y CCIE [Cisco Certified Internetwork Expert] ya que la interfaz gráfica de la herramienta es considerada intuitiva para el usuario.

Para lo referente a las tecnologías que soporta el software, el caso que presenta Djenane (2009) muestra la configuración de la red de Algerie Telecom para la simulación de VPN sobre MPLS usando IOS de Enrutadores Cisco, advirtiendo que, de no usar este tipo de dispositivos en la implementación real, el principio de funcionamiento es básicamente el mismo. La configuración de los dispositivos incluye los siguientes protocolos RIP, BGP, OSPF, entre otros. Además de esto, Tamanna y Fatema (2017) abordaron un problema sobre la conectividad de los proveedores de servicios de red de la nueva era usando GNS3, para esto detallan y proveen una posible solución a la problemática de interconectar diferentes sitios de una misma compañía, dispersos alrededor del mundo, mediante una red IP de un proveedor de servicios, usando el protocolo MPLS VPN con características GRE multipunto. Este simulador está enfocado a diseñar, construir y probar ambientes virtuales para la industria y la educación. Como principal característica y diferenciador de esta herramienta se resalta la capacidad de simular y emular dispositivos de red reales, lo cual permite configurar y desplegar redes de comunicaciones en un computador, cabe anotar que, dado que estos dispositivos ejecutan los sistemas operativos de los dispositivos reales, una de las limitantes a la hora de usar GNS3 es la cantidad de recursos de hardware disponibles en el equipo de simulación.

\section{Netsim Tetcos}

NetSim es un software para modelado y simulación de redes y protocolos enfocado a la investigación y desarrollo en redes, también usado en aplicaciones de defensa. Actualmente es comercial y se puede encontrar en tres versiones: profesional, estándar y académica. Según lo especificado en el sitio web oficial, 
las funcionalidades de las licencias estándar y académica están limitadas a investigación y experimentación en laboratorios de redes, mientras que la licencia profesional es adecuada para clientes comerciales. Esta herramienta se caracteriza por su alta usabilidad, debido a las funcionalidades otorgadas por sus dos interfaces de acceso: una gráfica y otra de comandos. La interfaz gráfica (GUI) de NetSim permite configurar los diferentes escenarios, ejecutar la simulación de eventos discretos, controlar y visualizar las simulaciones de forma animada, y analizar los resultados obtenidos en las simulaciones (Sundararajan y Arumugam, 2015). Por otro lado, su consola de comandos de línea (CLI) permite establecer el escenario de simulación mediante archivos de configuración XML y ejecutar simulaciones de eventos discretos. Sus capacidades han permitido lograr simulaciones bastante precisas y con resultados importantes en el ámbito científico; en (Johnson, 1999) se muestra como NetSim es usado para simular redes HF de tercera generación con diferentes topologías y cargas de trabajo. Adicionalmente, Sundararajan y Arumugam (2015) lo usaron para simular un sistema de detección de intrusos en una red de sensores inalámbricos que usa LEACH como protocolo de enrutamiento para su operación. Además, soporta una amplia gama de tecnologías, tales como: WiFi, WSN, OSPF, BGP, TCP, UDP, MANET, Wimax, GSM y CDMA, Zigbee, LTE y LTE avanzado (Singh et al. 2017). Uno de los aspectos limitantes de esta herramienta es su compatibilidad entre plataformas, ya que solo está disponible para sistemas operativos Windows, tal como se especifica en la página web oficial de NetSim.

Finalmente, es importante resaltar el valor agregado y el uso o enfoque que se le da a este simulador. Netsim Tetcos cuenta con una interfaz gráfica que permite diseñar y modelar entornos de red con gran nivel de detalle, este simulador ofrece tres tipos de licencia, las cuales ofrecen diferentes protocolos y aplicaciones para labores académicas o investigativas de simulación. Un elemento importante a resaltar es la gran cantidad de aplicaciones disponibles en su versión profesional, las cuales son redes celulares, de internet de las cosas, de sensores inalámbricos, de área personal, de radio cognitiva, de 5G, vehiculares y enlaces de radio de uso militar.

\section{Simulador Real}

REAL es un simulador de red originalmente diseñado para estudiar el comportamiento dinámico de esquemas de control de flujo y congestión en redes de datos con paquetes conmutados. Cabe aclarar que las simulaciones se realizan creando segmentos de código e integrándolos con los módulos que provee el simulador, lo cual hace que la curva de aprendizaje sea alta. Según el sitio web oficial, dichos módulos están construidos en el lenguaje de programación $\mathrm{C}$ y tienen una extensión de aproximadamente 15000 líneas de código. Este simulador se encuentra disponible para plataformas Unix, SunOS, Solaris, IRIX, BSD4.3, Ultrix, UMIPS. En versiones anteriores, su interfaz gráfica permitía representar el escenario de red mediante un grafo y configurar los escenarios de simulación mediante el ratón y los menús de la interfaz. En su versión 5.0 este simulador incluyó una interfaz gráfica escrita en Java, que permite construir rápidamente escenarios de simulación con una interfaz de apuntar y "hacer" clic. En cuanto a las tecnologías y protocolos que permite simular esta herramienta, REAL provee 30 módulos que permiten emular las opciones de protocolos de transporte, TCP, XNS, Ventanas deslizantes, tiempos muertos, adición de fuentes JK y DEC; y múltiples algoritmos de programación como FCFS, FQ y DEC (Keshav, 1997). Es importante mencionar como valor agregado y principal aplicación que esta herramienta fue diseñada para el estudio o evaluación del comportamiento dinámico de los esquemas de control de congestión y flujo, en redes de datos conmutadas por paquetes, por lo cual es ampliamente usada en los campos de investigación y educación.

Los resultados de la simulación se obtienen en un formato de reporte que incluye un registro de información por cada intervalo de tiempo, compuesto de ocho valores: 1) el número del nodo de la red; 2) el nombre de la función que se ejecutó en cada nodo; 3) el número del Gateway que se encarga de reportar los tiempos de retraso y la política de programación; 4) el número de paquetes transmitidos; 5) el número máximo, aproximado y mínimo de tiempos de encolamiento, y en paréntesis cuadrados el número de paquetes encolados por cada conversación; 6) el número de paquetes de esa fuente que fueron eliminados por pasarelas intermedias; 7) el número de paquetes que fueron retransmitidos; y 8) los valores mínimo, promedio y máximo de tiempo de viaje de cada paquete de esa fuente, contado desde el tiempo en que el primer bit del paquete es ubicado en la línea hasta el momento en que llega el ultimo bit del paquete de confirmación (Keshav, 1998).

\section{Qualnet}

Según la documentación que provee el fabricante, este simulador está enfocada para científicos, ingenieros y planeadores de red, que deseen crear modelos virtuales para todo tipo de red y de información. Qualnet permite diseñar escenarios para simulaciones de investigación en protocolos y tecnologías de ondas, diseño de redes y optimización de arquitecturas, modelamiento de interferencia y propagación de radio frecuencia, identificación de problemas de comunicación, preparación de respuestas a desastres y análisis de desempeño de aplicaciones, entre otras. Para usar este simulador los requerimientos hardware recomendados son: mínimo 4GB de memoria RAM, mínimo de 5GB de espacio en disco duro y una tarjeta gráfica de al menos 
2GB, que soporte aceleración 3D. Su licencia es de uso comercial y su curva de aprendizaje media, ya que su interfaz dispone las herramientas y opciones necesarias para su utilización. Actualmente está disponible para Windows 7 y Windows 8 , en versiones de 64bit, también para sistemas operativos Linux con arquitectura x86, tales como CentOS 6.6, Red Hat Enterprise Linux (RHEL) y Ubuntu 14.04 LTS.

Aprovechando las capacidades de esta herramienta, se encontró que se han realizado múltiples simulaciones de redes inalámbricas. (Bhat.M et al. 2011), simularon protocolos de enrutamiento en redes MANET para estudiar su desempeño. También, Mittal y Kaur (2009) simularon múltiples escenarios con la finalidad de estudiar el desempeño de algunas estrategias de enrutamiento en redes MANET. Sharma y Gupta (2009) los usaron para simular brechas de seguridad del protocolo AODV y estudiar los ataques de hoyos negros en redes MANET. Qualnet se compone de cinco elementos, de los cuales tres son interfaces graficas que permiten diseñar, analizar y visualizar la simulación: el primer componente es Qualnet Architect, el cual permite diseñar el escenario (configurar el terreno, las conexiones de red, patrones de movilidad de usuarios inalámbricos, entre otros patrones funcionales de cada nodo de red); el segundo permite graficar las estadísticas y métricas recolectadas durante la simulación y obtener un reporte, en formato CSV; y el ultimo componente grafico es una herramienta que permite obtener una representación visual de la ruta de los paquetes generados durante la simulación del escenario de red, dicha ruta se presenta en un archivo de formato XML que contiene información sobre los paquetes a medida que se mueven hacia arriba y hacia abajo de la pila de los protocolos. Las principales aplicaciones de este, son el modelamiento de interferencia y propagación de radio frecuencia, la identificación de problemas de comunicación, preparación de respuestas a desastres y análisis de desempeño de aplicaciones. El elemento diferenciador de Qualnet se da cuando el ambiente a simular requiere análisis de información asociada a una red, ya que en este caso las redes simuladas consideran detalles geológicos como detalles del terreno, efectos de la propagación de canal, subredes alámbricas, entre otras.

\section{Simulador J-sim}

J-SIM fue desarrollado por un equipo del laboratorio de computación distribuida en tiempo real (DRCL). En un ambiente de simulación y animación basado en Java que soporta la animación basada en Web. Este proyecto fue patrocinado por la Fundación Nacional para la Ciencia [National Science Foundation, NSF], el programa de modelado y simulación de redes DARPA, la Universidad del Estado de Ohio y la Universidad de Illinois en Urbana-Champaign. Su código está disponible en Internet y es de libre uso. Lo anterior según el sitio oficial del proyecto. Este simulador ha sido usado para emular ambientes de red inalámbricas con múltiples especificaciones y gran capacidad para establecer detalles. Sobeih et al. (2006) compararon J-Sim con otros dos simuladores (NS-2 y SSFNet) para evaluar su desempeño, tiempo de simulación y uso de memoria. J-Sim obtuvo los resultados más favorables con un menor uso de memoria y mayor capacidad para escalar, gracias al recolector de basura usado en Java para recuperar la memoria no utilizada. Sobeih et al. (2007) usaron J-Sim para simulación y comprobación de modelos de protocolos de red, además se comprobó el modelo Ad hoc On-Demand Distance Vector [AODV], el cual se caracteriza por su complejidad y por ser ampliamente usado.

En la actualidad, el sitio oficial de J-Sim provee los archivos de instalación para sistemas operativos Unix/Linux y Windows. Para ejecutar este simulador se requiere tener instalada la máquina virtual de Java [Java Virtual Machine, JVM]. La última versión liberada de J-Sim demanda bajos recursos, tales como mínimo 128MB de memoria RAM y procesadores Pentium II o Celeron 466; esta última versión, J-Sim 1.3, fue liberada y publicada el 21 de Julio de 2008. El sitio web del proyecto contrasta, y detalla, algunas características de JSim frente a otros simuladores; en cuanto a las capacidades de simulación de J-Sim encontramos protocolos y modelos de red como IP, TCP, UDP, OSPFv2 y Protocolos de enrutamiento multidifusión (DVMRP, MOSPF, CBT). Inicialmente J-Sim contaba con su propia herramienta de lenguaje por comandos [Tool Command Language, TCL], en la cual mediante comandos se podían diseñar, simular y analizar los diferentes escenarios. Según la documentación oficial, J-Sim puede integrarse con gEditor, un editor gráfico para diseñar y simular escenarios de red, este editor usa XML como lenguaje para modelar los diferentes componentes de red. Lo anterior deja ver que su curva de aprendizaje es alta, ya que se requieren conocimientos avanzados en lenguaje TCL, Java, redes y programación para poder interactuar con la herramienta.

Finalmente, como elemento diferenciador $\mathrm{J}$-sim resalta que es un simulador que no requiere alto poder computacional y dado que se ejecuta en la máquina virtual de Java puede ejecutar diferentes simulaciones con un menor uso de memoria y mayor capacidad para escalar, esto gracias al recolector de basura usado en Java para recuperar la memoria no utilizada. Además de esto, dado que es un software de código abierto y en constante reforma por su comunidad, no tiene un foco principal de aplicación y deja el camino para que los mismos usuarios puedan generar módulos de procesamiento y análisis. 


\section{DISCUSIÓN}

Como se anticipó, la Tabla 1 corresponde a una matriz que relaciona cada una de las herramientas con cada uno de los parámetros de selección. Para la realización de proyectos donde se involucre el uso de herramientas de simulación de red, es de gran importancia invertir tiempo y estudio en la elección de la herramienta adecuada, para ello, se debe tener claro cuáles son las características más relevantes que dicho software debe proporcionar, ya que de esto depende la efectiva culminación y pruebas a realizar en el proyecto. De este modo, la Tabla 1 muestra la comparación entre diferentes herramientas de simulación, teniendo en cuenta los parámetros definidos, que permiten guiar al usuario en el proceso de elección, con el fin de tomar la decisión que más se ajuste a sus requerimientos. Un ejemplo de lo anterior sería, un usuario que necesitara analizar parámetros de calidad de servicio y tipos de algoritmos de encolamiento sobre una arquitectura de red congestionada, en ese caso la Tabla 1, los criterios definidos y las características de las herramientas planteadas en este documento sugieren a OPNET y OMNET++ como las herramientas de simulación adecuadas. Pero aunque OPNET sea una herramienta mucho más robusta en aspectos de configuración (Tráfico que permite modelar), su licencia es costosa y su adquisición en algunos grupos de investigación o universidades no es viable económicamente. Mientras que OMNET++ al poseer licencia libre hace que sea mucho más fácil de conseguir en ambientes académicos o investigativos.

Aunque todos los parámetros presentados en la Tabla 1 son importantes para el proceso de elección, el tipo de licencia de las herramientas es determinante, puesto que aquellas con licencia libre, generalmente son la primera opción para los usuarios que no tienen la posibilidad de costear una. Sin embargo, el hecho de que una herramienta posea licencia libre debe contrastarse con la documentación y soporte de la misma, ya que de este modo se tendría claro el "apoyo y el alcance", para la revisión de errores o problemas a los que otros autores se han enfrentado y corregido. Diferente a lo que ocurre con los simuladores de licencia comercial, que brindan el soporte durante el tiempo de validez de la licencia. Otro parámetro importante para tener en cuenta es el uso específico que se le ha dado a cada simulador, a pesar de que en su mayoría son capaces de simular diferentes tipos de redes, las referencias en un tema específico hacen que la cantidad de información, módulos, y desarrollo en el tema sea mayor, lo cual permite establecer en un primer análisis si la herramienta se ajusta a los requerimientos del usuario o no. En cuanto a la generación y presentación de resultados estadísticos en una herramienta de simulación es vital, ya que, con base en ellos, el investigador puede hacer aportes a la comunidad investigativa y/o académica. Y algunas herramientas poseen mejores especificaciones que otras en este aspecto, lo cual permite generar este tipo de resultados sin necesidad de usar aplicaciones externas o tener que realizar procesamientos extensos para la presentación de datos producto de las simulaciones.

Finalmente, se recomienda que el usuario inicie la selección de la herramienta de simulación, filtrando según el tipo de licencia que desea usar y la curva de aprendizaje que desea invertir, ya que estos dos factores permiten agrupar la mayoría de las herramientas; después, se debe comenzar a filtrar teniendo en cuenta el contexto de la simulación, es decir tecnologías de nivel 2 y 3 que soporta, y tráfico que permite modelar, de esta forma se limitan rápidamente las posibles opciones y se asegura la elección según la necesidad de cada usuario. Por otro lado, se considera que el tipo de plataforma que soporta la herramienta es una variable de baja influencia, ya que actualmente con soluciones de virtualización es posible ejecutar cualquier herramienta sin restringirse a un único sistema operativo.

Tabla 1: Parametrización de las herramientas de simulación presentadas en el artículo.

\begin{tabular}{|l|l|l|l|l|l|l|l|l|}
\hline & \multicolumn{1}{|c|}{$\begin{array}{l}\text { Uso } \\
\text { investigativo }\end{array}$} & \multicolumn{1}{|c|}{$\begin{array}{c}\text { Tipo de } \\
\text { Licencia }\end{array}$} & $\begin{array}{c}\text { Curva de } \\
\text { Aprendizaje }\end{array}$ & $\begin{array}{c}\text { Plataformas } \\
\text { que Soportan }\end{array}$ & $\begin{array}{c}\text { Interfaz } \\
\text { Gráfica de } \\
\text { Herramienta }\end{array}$ & $\begin{array}{c}\text { Graficación } \\
\text { de } \\
\text { Resultados }\end{array}$ & $\begin{array}{l}\text { Tecnologías } \\
\text { de Nivel 2 y } \\
\text { Nivel 3 }\end{array}$ & $\begin{array}{c}\text { Tráfico } \\
\text { permitido }\end{array}$ \\
\hline OPNET & Alto & Comercial & Alto & Windows, Unix & Alto & Buena & Alto & Alto \\
\hline OMNET & Alto & Libre & Alto & Windows, Unix & Medio & Aceptable & Alto & Medio \\
\hline NS-3 & Medio & Libre & Alto & $\begin{array}{l}\text { Windows, } \\
\text { MAC, Unix }\end{array}$ & Medio & Aceptable & Medio & Medio \\
\hline GNS3 & Bajo & $\begin{array}{l}\text { Libre/ } \\
\text { Comercial }\end{array}$ & Bajo & $\begin{array}{l}\text { Linux, } \\
\text { MAC,Windows }\end{array}$ & Alto & limitada & Bajo & Nulo \\
\hline NS-2 & Alto & Libre & Alto & $\begin{array}{l}\text { Windows, } \\
\text { MAC, Unix. }\end{array}$ & Bajo & No tiene & Alto & Alto \\
\hline
\end{tabular}


Tabla 1 (continuación)

\begin{tabular}{|l|l|l|l|l|l|l|l|l|}
\hline EstiNet & Alto & Comercial & Alto & Linux & Alto & Aceptable & Alto & Alto \\
\hline $\begin{array}{l}\text { NETSIM } \\
\text { TETCOS }\end{array}$ & Alto & Comercial & Alto & Windows & Alto & Aceptable & Alto & Alto \\
\hline REAL & Alto & Libre & Alto & Unix, Solaris & Media & Limitado & Medio & Medio \\
\hline QUALNET & Alto & Comercial & Medio & $\begin{array}{l}\text { Windows, } \\
\text { Linux }\end{array}$ & Alto & Buena & Alto & Alto \\
\hline J-SIM & Alto & Libre & Alto & $\begin{array}{l}\text { Windows, } \\
\text { Linux }\end{array}$ & Media & Limitado & Medio & Medio \\
\hline
\end{tabular}

\section{CONCLUSIONES}

Las herramientas de simulación son pilar fundamental tanto en los procesos enseñanza-aprendizaje como en el campo investigativo. Es por esta razón que elegir una herramienta de simulación requiere un análisis detallado, donde los primeros parámetros que se tienen en cuenta para su elección son: tipo de licencia y la curva de aprendizaje, posterior a esta elección se analiza las tecnologías de nivel 2 y nivel 3 que soporta. En ambientes académicos, la curva de aprendizaje juega un papel fundamental. Debido a que dependiendo de la madurez del estudiante la herramienta le debe facilitar los procesos de enseñanza-aprendizaje, es por esta razón que para cursos introductorios en telecomunicaciones la herramienta GNS3 es la más adecuada.

Por su parte, en ambientes investigativos la situación cambia drásticamente, porque primero se debe definir el alcance de lo que se desea simular y con base en esto, empezar la búsqueda de la herramienta. Considerando que se debe incluir en el cronograma del proyecto, el tiempo para aprender a manejar la herramienta (curva de aprendizaje) y mientras se está en este proceso se debe analizar que tecnologías de nivel 2 y 3 soporta, como también el tráfico que permite modelar. Esto debido a que, algunas herramientas no describen detalladamente las tecnologías de nivel 2 y 3 que soportan como también el tráfico que permiten modelar. Esto se descubre directamente trabajando con la herramienta en casos específicos. Para solucionar los dos aspectos antes mencionados, herramientas como NS-2 y NS-3 son adecuadas, ya que el tráfico a modelar, el usuario lo puede programar de acuerdo a sus necesidades, pero inversión en tiempo es alta.

Hace algunos años la plataforma o sistema operativo en el cual se debía instalar la plataforma influía en su elección, pero actualmente es una variable de baja influencia debido a que con esquemas de virtualización se pueden ejecutar varios sistemas operativos en una misma máquina. $Y$ adicionalmente adquiriendo servicios en la nube trabajar con este tipo de herramientas es mucho más sencillo y más productivo. Para finalizar el cuadro comparativo presentado en la tabla 1, permite tanto reducir los tiempos en la selección y elección de una herramienta de simulación, ya el cuadro comparativo presenta de forma concreta los parámetros de mayor influencia entre los simuladores de red analizados, lo cual permite a investigadores, profesores, estudiantes y demás interesados, escoger rápidamente y en menor tiempo la solución tecnológica que más se ajuste a sus necesidades.

\section{REFERENCIAS}

Bateman, M. y S. Bhatti, TCP Testing: How Well Does ns2 Match Reality?, 24th IEEE International Conference on Advanced Information Networking and Applications, 276-284, Perth W.A. - Australia, 20-23 de Abril (2010)

Batish, S. y A. Dhiman, Comparative Study of AODV, DSDV and DSR Routing Protocols in Vehicular Network Using EstiNet Simulator, International Journal of Scientific and Engineering Research, 3(6), 1-5 (2012)

Ben Fraj, R., V. Beroulle, N. Fourty y A. Meddeb, An Evaluation of UHF RFID Anti-Collision Protocols with NS2, 9th IFIP International Conference on New Technologies, Mobility and Security, 47-56, Paris - Francia, 26-28 de Febrero (2018)

Bhat, S., D. Shwetha y J. Devaraju, A Performance Study of Proactive, Reactive and Hybrid Routing Protocols using Qualnet Simulator, doi: 10.5120/3385-4696, International Journal of Computer Applications, 28(5), 10-17 (2011)

Brugge, J., L., Paquereau y P.E. Heegaard, Experience Report on Implementing and Simulating a Routing Protocol in NS-2 and NS-3, 2nd International Conference on Advances in System Simulation, 88-93, Niza, Francia, 22-27 de Agosto (2010)

Chen, Y.C., S.S. Li y K.T. Chen, An Efficient Source Allocation Approach for QoS Support in P2PTV Systems, In 2010 IEEE 34th Annual Computer Software and Applications Conference, 509-514, Seoul-South Korea, 30 de Diciembre (2010)

Cuéllar, J.C., Analysis of Quality of Service Parameters Using a Simulation Tool, ISSN: 1909-8367, Entre Ciencia e Ingeniería, 5(9), 120-131 (2011)

Cuellar, J.C., J.H. Ortiz y J.L. Arciniegas, Clasificación y Análisis de Métodos para medir Calidad de la Experiencia del Servicio de Televisión sobre Protocolo IP (IPTV), Información Tecnológica, 25(5), 121-128 (2014) 
Djenane, N., A. Benaouda y S. Harous, Simulation of a VPN Implementation based on MPLS Protocol, A Case Study: VPN-MPLS for MSN-AT, Proceedings of the 7th International Conference on Advances in Mobile Computing and Multimedia, 589-593, Kuala Lumpur-Malaysia, 14-16 de Diciembre (2009)

Fan, L. y L. Taoshen, Implementation and Performance Analyses of Anycast QoS Routing Algorithm Based on Genetic Algorithm in NS2, doi: 10.1109 / ICIC.2009.298, IEEE 2009 Second International Conference on Information and Computing Science, 04, 368-371 (2009)

García, A., M. Escobar, A. Navarro y A. Vásquez, Método de Evaluación y Selección de Herramientas de Simulación de Redes, Sistemas y Telemática, 9(16), 55-71 (2011)

García, R.C, O.J. Salcedo, D.A. López y L.F. Pedraza, Evaluación de la calidad del servicio para voz sobre protocolo de internet (VOIP) en redes Wimax sobre ambientes IP/MPLS, Información Tecnológica, 25(2), 24-38 (2014)

García, N.Y., Vera, N.E. y D.A. López, Análisis del Funcionamiento del Algoritmo de Balanceo de Carga LCM en Redes de Conmutación de Etiquetas Multiprotocolo Generalizado (GMPLS), Información Tecnológica, 26(1), 41-54 (2015)

Goldstein, C., S. Leisten, K. Stark y A. Tickle, Using a Network Simulation Tool to Engage Students in Active Learning Enhances Their Understanding of Complex Data Communications Concepts. In Proceedings of the 7th Australasian Conference on Computing Education, 223-228, Newcastle - Australia, 01 de Enero (2005)

Hammoodi, I.S., B.G. Stewart, A. Kocian. y S.G. McMeekin, A Comprehensive Performance Study of OPNET Modeler for ZigBee Wireless Sensor Networks, In 2009 Third International Conference on Next Generation Mobile Applications, Services and Technologies, 357-362, Cardiff - Wales, 15-18 de Septiembre (2009)

Henderson, T.R., S. Roy, S. Floyd y G. Riley, Ns-3 Project Goals, doi: 10.1145/1190455.1190468, Proceeding from the 2006 Workshop on Ns-2: The IP Network Simulator, 6, 13 (2006)

Johnson, E.E., Simulation Results for Third-generation HF Automatic Link Establishment, IEEE Military Communications Conference Proceedings, 984-988 (1999)

Keshav, S., REAL: A Network Simulator, Technical Report 88/472, Dept. of Computer Science, UC Berkeley, University of California - Berkeley (1988)

Kitanov, S. y T. Janevski, Modeling and Analyzing LTE Networks with EstiNet Network Simulator and Emulator, In proceedings - International Scientific Conference on Information, Communication and Energy Systems and Technologies, 95-98, Ohrid - Macedonia, 26-29 de Junio (2013)

Kucerak, J. y P. Chlumsky, SIP protocol Model for Omnet ++, doi: 10.15598/aeee.v14i3.1502, Advances in Electrical and Electronic Engineering, 14(3), 274-278, (2016)

Kulgachev, V. y H. Jasani, 802.11 Networks Performance Evaluation using OPNET. In Proceedings of the 2010 ACM conference on Information technology education, 149-152, Michigan - USA, 07 - 09 de Octubre (2010)

Lessmann, J., P. Janacik, L. Lachev y D. Orfanus, Comparative Study of Wireless Network Simulators, Seventh International Conference on Networking, 517-523, Cancun, Mexico, 13-18 de Abril (2008)

Li, J., Y. Zhang, J. Zhao, Y. Wang, X. Ma y W. Wei, NS-2 Simulation of Vanet for Safety applications: issues and solutions. Proceedings of the 8th International Conference on Computer Modeling and Simulation, 67-72, Canberra-Australia, 20-23 de Enero (2017)

López, D.A., N.Y. García y P.J. Figueroa, Estimación y optimización del balanceo de carga usando ingeniería de tráfico adaptativa mate en redes de conmutación de etiquetas multiprotocolo generalizado (GMPLS), Información Tecnológica, 25(2), 47-56 (2014)

Marques, E.M.D., R.A.S.A. Placido y P.N.M Sampaio, Visual Network Simulator (VNS): A GUI to QoS simulation for the ns-2 simulator, In 2009 IEEE/ACS International Conference on Computer Systems and Applications, 342-349, Rabat Marruecos, 10-13 de Mayo (2009)

Merzoug, S., O. Kazar y M. Derdour, Intelligent Strategy of Allocation resource for Cloud Datacenter Based on MAS \& CP approach, Proceedings of the International Conference on Computing for Engineering and Sciences, 50-55, IstanbulTurkey, 22-24 de Julio (2017)

Mittal, S. y P. Kaur, Performance Comparison of AODV, DSR and ZRP Routing Protocols in MANET's, International Conference on Advances in Computing, Control and Telecommunication Technologies, 165-168, Kerala - India, 28-29 de Diciembre (2009)

Morales, M., M.A. Calle, J.D. Tovar y J.C. Cuellar, Simulando con OMNET: Selección de la Herramienta y su Utilización, 1(1), 13-82, Universidad Icesi - Facultad de Ingeniería, Cali, Colombia (2013)

Pan, J. y R. Jain, A Survey of Network Simulation Tools: Current Status and Future Developments, Universidad de Washington, San Luis, EE. UU. (2008)

Perez-Hardy, S., The Use of Network Simulation to Enhance Network Curriculum. In Proceeding of the 4th conference on Information technology education, 93-95, Lafayette - USA, 16-18 de Octubre (2003)

Phillips, C., A Review of High Performance Simulation Tools and Modeling Concepts. In Recent Advances in Modeling and Simulation Tools for Communication Networks and Services, (1), 29-47, Springer, Boston, USA, (2007) 
Qun, Z.A. y W. Jun, Application of NS2 in Education of Computer Networks. In 2008 International Conference on Advanced Computer Theory and Engineering, 368-372, Phuket, Thailand, (2008)

Rhee, B., S. Cho, J. Xiansu. y S. Han, QoS-Aware Router Combining Features of Conventional Routing and Flow-Aware Routing Based on Resource Management over NGN, In 2009 International Conference on Advanced Information Networking and Applications Workshops, 413-418, Bradford - United Kingdom, 26-29 de Mayo (2009)

Sarkar, M. y G. Ramesh, An Algorithm to Enhance QoS for Streaming Video over WLANs, In World Congress on Engineering and Computer Science 2008, 76-85, San Francisco - USA, 22-24 de Octubre (2008)

Sharma, S. y R. Gupta, Simulation Study of Blackhole Attack in the Mobile Ad Hoc Networks, Journal of Engineering Science and Technology, 4(2), 243-250 (2009)

Shehab, A., M. Elhoseny, M.A. El Aziz y A.E. Hassanien, Efficient Schemes for Playout Latency Reduction in P2P-VoD Systems, Advances in Soft Computing and Machine Learning in Image Processing, (730), 477-495, Springer, Cham (2018)

Shehab, A., M. Elhoseny y A.E. Hassanien, An efficient Scheme for Video Delivery In Wireless Networks, Quantum Computing: An Environment for Intelligent Large Scale Real Application, (33), 207-225, Springer, Cham (2018)

Shin, J.Y., J.W. Jang y J.M. Kim, Result Based on NS2, Simulation and Emulation Verification. In 2009 International Conference on New Trends in Information and Service Science, 807-811, Beijing - China, 30 de Junio - 2 de Julio (2009)

Singh, G., A.K. Cheema y N. Kapoor, Performance Evaluation of Routing Protocol in Internet of Things using Netsim, doi: 10.26483/ijarcs.v8i3.3114, International Journal of Advanced Research in Computer Science, 8(3), 856-859 (2017)

Sobeih, A. y J.C. Hou, A Simulation Framework for Sensor Networks in J-Sim, Simulation Symposium, 175-187, San Diego - USA, 4-6 de Abril (2006)

Sobeih, A., M. Viswanathan, D. Marinov y J.C. Hou, J-Sim: An Integrated Environment for Simulation and Model Checking of Network Protocols, 07' IEEE International Parallel and Distributed Processing Symposium, 1-6, Roma - Italia, 26-30 de Marzo (2007)

Sundararajan, R.K. y U. Arumugam, Intrusion Detection Algorithm for Mitigating Sinkhole Attack on LEACH Protocol in Wireless Sensor Networks, doi: 10.1155/2015/203814, Journal of Sensors, 2015, 1-12 (2015)

Tamanna, T. y T. Fatema, MPLS VPN Over mGRE Design and Implementation for a Service Provider's Network Using GNS3 Simulator, International Conference on Wireless Communications, Signal Processing and Networking (WiSPNET), 2339-2342, Chennai-India, 22-24 de Marzo (2017)

Varga, A., The OMNET++ Discrete Event Simulation System, Proceedings of the European Simulation Multiconference, 1-7, Praga - Republica Checa, 6-9 de Junio (2001)

Wang, A. y Z. Liu, Analysis and Utilizing of the Error Models in Network Education with NS3. In 2nd International Workshop on Education Technology and Computer Science, 15-18, Wuhan-China, 6-7 de Marzo (2010)

Wang, S.Y., C.L. Chou y C.M. Yang, EstiNet openflow network simulator and emulator, doi: 10.1109/MCOM.2013.6588659, IEEE Communications Magazine, 51(9), 110 - 117 (2013)

Weingartner, E., H.V. Lehn y K. Wehrle, A Performance Comparison of Recent Network Simulators, Proceedings of the 2009 IEEE International Conference on Communications, 1287-1291, Dresden-Germany, 14-18 de Junio (2009)

Xia, J.B., M.H. Li y L.J. Wan, Research on MPLS VPN Networking Application Based on OPNET, In 2008 International Symposium on Information Science and Engineering, 404-408, Shangai-China, 20-22 Diciembre (2008)

Zhu, W., T. Dreibholz, E.P. Rathgeb y X. Zhou, A Scalable QoS Device for Broadband Access to Multimedia Services, 2008 Second International Conference on Future Generation Communication and Networking, 343-348, Hainan IslandChina, 13-15 de Diciembre (2008) 